\title{
On conforming tetrahedralizations of prismatic partitions
}

\author{
Sergey Korotov ${ }^{1,2}$, Michal Křížek ${ }^{3}$
}

October 11, 2011

${ }^{1}$ BCAM - Basque Center for Applied Mathematics Bizkaia Technology Park, Building 500, E-48160 Derio

Basque Country, Spain

e-mail: korotov@bcamath.org

${ }^{2}$ IKERBASQUE, Basque Foundation for Science

E-48011 Bilbao, Spain

${ }^{3}$ Institute of Mathematics, Academy of Sciences

Žitná 25, CZ-115 67 Prague 1, Czech Republic

e-mail: krizek@math.cas.cz

\begin{abstract}
We present an algorithm for conform (face-to-face) subdividing prismatic partitions into tetrahedra. This algorithm can be used in the finite element calculations and analysis.
\end{abstract}

Keywords: finite element method, prismatic element, tetrahedral mesh, linear elements

Mathematical Subject Classification: 65N50, 51M20

\section{Introduction}

Tetrahedral, prismatic, pyramidal, or block elements are usually used in finite element approximations of various engineering three-dimensional problems. Therefore, a natural question arises which of these elements are the most suitable for a particular problem in a given domain (cf. [1], [3], [7], [8]). The use of several types of elements enables us to compare the influence of the space discretization on the finite element solution.

However, among various types of elements, tetrahedral elements are the most popular for many reasons. For instance, if block trilinear elements are employed, the discrete maximum principle need not be satisfied (see [6], [5]). But if each block element is divided into 6 nonobtuse tetrahedra such that all of them contain the spatial diagonal, then the stiffness matrix associated to linear elements has the same size and the discrete maximum principle is fulfilled for a large class of nonlinear elliptic problems (see [4]). Moreover, the stiffness matrix associated with linear finite elements has less nonzero diagonals than 
that one for trilinear block or triangular prism elements. Another important reason for the use of tetrahedral elements is their flexibility to describe complicated boundaries.

In this work we show how to conformly (face-to-face) subdivide the given prismatic partition into tetrahedra as local subdivisions of prisms into tetrahedra cannot be done independently from each other in order to get a face-to-face tetrahedralization.

\section{Subdivision of prismatic partitions into tetrahedra}

By prism (or more precisely triangular prism) we shall mean a prism with two parallel triangular faces and three rectangular faces.

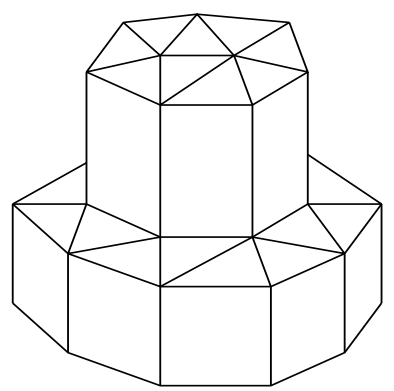

Figure 1: Partition into prisms.

In what follows we consider only bounded polyhedra $\bar{\Omega} \subset R^{3}$ which can be decomposed into prisms. Let $\mathcal{P}_{h}$ be a face-to-face partition of one such polyhedron into prisms (see Figure 1). Here $h$ stands for the usual discretization parameter, i.e., the maximum diameter of all prisms from $\mathcal{P}_{h}$.

Throughout the paper we will always consider only face-to-face partitions, and therefore, the notion "face-to-face" will be sometimes omitted. It is evident from the definition of a prism that any partition $\mathcal{P}_{h}$ into prisms consists of parallel layers of prisms. In the following we may suppose these layers to be horizontal and we call the bottom plane of a layer its base and the triangular face of a prism we call the base triangle of the prism.

We shall consider such tetrahedralizations of $\mathcal{P}_{h}$ that the trianglular faces of the prisms are not cut. Hence, the different layers of prisms can be subdivided independently into tetrahedra and these altogether provide us with a conforming tetrahedral mesh over $\bar{\Omega}$.

We shall subdivide each prism into three tetrahedra as marked in Figure 2 (left). We see that its rectangular faces are divided by diagonals into triangles and these diagonals determine three tetrahedra in the subdivision. However, these diagonals cannot be chosen arbitrarily. In Figure 2 (right) we observe a division of three rectangular faces of a prism which does not correspond to any partition of the prism into tetrahedra. Therefore, we have to divide rectangular faces in the whole partition carefully.

In the next theorem we show how to practically construct from a given prismatic partition $\mathcal{P}_{h}$ a face-to-face tetrahedralization, thus avoiding the situation illustrated in Figure 2 (right) (or its mirror image) when dividing rectangular faces by diagonals.

Theorem 2.1 For any conforming partition into prisms there exists a face-to-face subdivision into tetrahedra. 

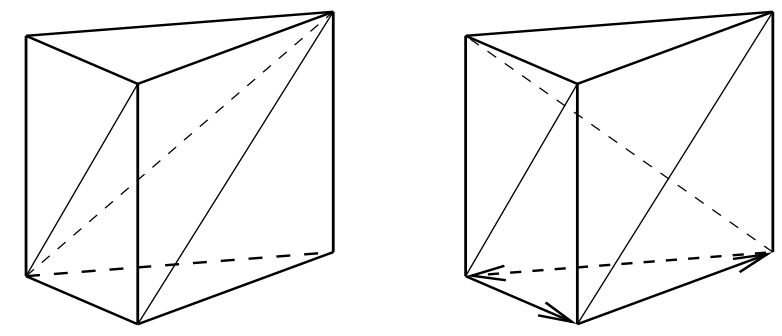

Figure 2: Two subdivisions of rectangular faces of a prism.

P r o o f : From the beginning of this section we know that any partition of $\mathcal{P}_{h}$ into prisms consists of parallel layers which can be tetrahedralized independently (see Figure 1). Consider one of such layers supposed to be horizontal and let $\mathcal{T}_{h}$ be the triangulation of its base corresponding to the partition $\mathcal{P}_{h}$. Take an arbitrary vector $\vec{v} \neq 0$ in the plane containing the triangulation $\mathcal{T}_{h}$ (for instance $\vec{v}=(1,0,0)$ ). Now we define the orientation $\vec{e}$ of each edge $e$ of the triangulation $\mathcal{T}_{h}$ such that

$$
(\vec{v}, \vec{e}) \geq 0 .
$$

If an edge $e$ is perpendicular to $\vec{v}$, we may take an arbitrary orientation of $\vec{e}$. In this way we get the planar digraph $G_{h}=(N, E)$, where $N$ is the set of nodes and $E$ is the set of the directed edges.

It is clear that $G_{h}$ does not contain a directed circle of which edges form a triangle of $\mathcal{T}_{h}$. Indeed, if, on the contrary, $\vec{e}_{1}, \vec{e}_{2}, \vec{e}_{3}$ form a circle then $\vec{e}_{1}+\vec{e}_{2}+\vec{e}_{3}=\overrightarrow{0}$. Taking the scalar product of both sides by $\vec{v}$ and using (1), we get that $v$ is perpendicular to the triangle with side vectors $\vec{e}_{1}, \vec{e}_{2}, \vec{e}_{3}$, which is a contradiction.

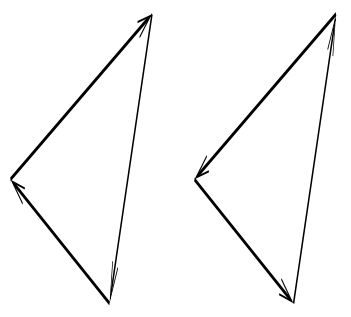

Figure 3: Non-allowed edge orientations.

Remark 2.1 Non-allowed edge orientations are sketched in Figure 3. In Figure 4 we see a partition of the prism $A B C A^{\prime} B^{\prime} C^{\prime}$ into three tetrahedra different from that in the left part of Figure 2.

We demonstrate now that the tetrahedral mesh generated as above is regular whenever the original prismatic mesh is regular as well.

Definition 2.1 A family of partitions $\mathcal{F}=\left\{\mathcal{T}_{h}\right\}_{h \rightarrow 0}$ of a polyhedron $\bar{\Omega}$ into convex elements is said to be regular (strongly regular) if there exists a constant $\kappa>0$ such that for any partition $\mathcal{T}_{h} \in \mathcal{F}$ and any element $T \in \mathcal{T}_{h}$ we have

$$
\kappa h_{T}^{3} \leq \text { meas } T \quad\left(\kappa h^{3} \leq \text { meas } T\right),
$$

where $h_{T}=\operatorname{diam} T$. 


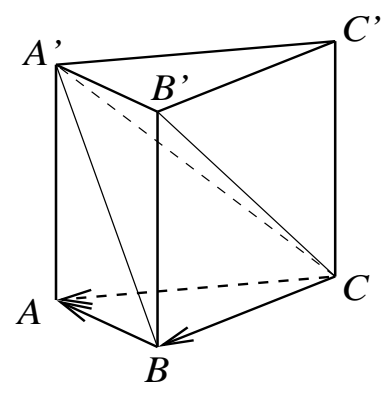

Figure 4: A possible orientation of edges of a triangular base that leads to a partition into three tetrahedra.

Remark 2.2 The above definition is equivalent to the inscribed ball condition (see, e.g., [2, Sec. 16]) which is more complicated. Note also that the regularity of a family of partitions into prisms is equivalent to Zlámal's minimum angle condition [2, p. 128] applied to triangular bases of all prisms provided the height of all prisms is proportional to $h$.

Theorem 2.2 If a family of partitions $\left\{\mathcal{P}_{h}\right\}_{h \rightarrow 0}$ of a polyhedron $\bar{\Omega}$ into prisms is regular (strongly regular) then the associated family of partitions $\left\{\mathcal{T}_{h}\right\}_{h \rightarrow 0}$ into tetrahedra is also regular (strongly regular).

$\mathbf{P} \mathbf{r}$ o o f : It is evident that the volume of each of the three tetrahedra from Figure 2 (left) is equal to the one third of the volume of the prism. Therefore, if inequalities (2) hold for prisms, then the same relations hold also for tetrahedra with another constant $\kappa^{\prime}=\kappa / 3$.

Remark 2.3 Assume that a family of partitions of $\bar{\Omega}$ into prisms is regular and that $\Omega$ has Lipschitz boundary. Then by Theorem 2.2 the optimal interpolation properties of tetrahedral elements in Sobolev space norms are satisfied.

Acknowledgement: This work was supported by Grant MTM2011-24766 of the MICINN, Spain, and the Grant no. IAA 100190803 of the Grant Agency of the Academy of Sciences of the Czech Republic.

\section{References}

[1] T. Apel, N. Düvelmeyer. Transformation of hexahedral finite element meshes into tetrahedral meshes according to quality criteria. Preprint SFB393/03-09, Tech. Univ. Chemnitz, 2003, 1-12.

[2] P. G. Ciarlet. Basic error estimates for elliptic problems, Handbook of Numer. Anal., vol. II. (ed. P. G. Ciarlet, J. L. Lions). North-Holland, Amsterdam, 1991.

[3] A. Hannukainen, S. Korotov, T. Vejchodský. Discrete maximum principle for FE solutions of the diffusion-reaction problem on prismatic meshes. J. Comput. Appl. Math. 226 (2009), 275-287. 
[4] J. Karátson, S. Korotov, M. Křižek. On discrete maximum principles for nonlinear elliptic problems. Math. Comp. Simulation 76 (2007), 99-108.

[5] S. Korotov, T. Vejchodský. A comparison of simplicial and block finite elements. In: Proc. Eighth Europ. Conf. on Numerical Mathematics and Advanced Applications (ENUMATH2009), Uppsala, Sweden, (ed. by G. Kreiss et al.), Springer-Verlag, Heidelberg, 2010, 531-540.

[6] M. Křížek, Q. Lin. On diagonal dominance of stiffness matrices in 3D. East-West J. Numer. Math. 3 (1995), 59-69.

[7] M. Křížek, P. Neittaanmäki. Finite Element Approximation of Variational Problems and Applications. Pitman Monographs and Surveys in Pure and Applied Mathematics vol. 50, Longman Scientific \& Technical, 1990.

[8] L. Liu, K. B. Davies, K. Yuan, M. Křŕžek. On symmetric pyramidal finite elements. Dyn. Contin. Discrete Impuls. Syst., Ser. B, Appl. Algorithms 11 (2004), 213-227. 